\title{
A Community-Driven Roadmap for the Adoption of Safety Security and Rescue Robots
}

\author{
Robin Murphy and Alexander Kleiner
}

Linköping University Post Print

\section{- Tweet}

N.B.: When citing this work, cite the original article.

(C2013 IEEE. Personal use of this material is permitted. However, permission to reprint/republish this material for advertising or promotional purposes or for creating new collective works for resale or redistribution to servers or lists, or to reuse any copyrighted component of this work in other works must be obtained from the IEEE.

Robin Murphy and Alexander Kleiner, A Community-Driven Roadmap for the Adoption of Safety Security and Rescue Robots, 2013, 11th IEEE International Symposium on Safety, Security, and Rescue Robotics (SSRR 2013), 21-26 October 2013, Linköping, Sweden.

Postprint available at: Linköping University Electronic Press

http://urn.kb.se/resolve?urn=urn:nbn:se:liu:diva-100157 


\section{A Community-Driven Roadmap for the Adoption of Safety Security and Rescue Robots}

\author{
Robin R. Murphy \\ Center for Robot-Assisted Search and Rescue \\ Texas A\&M University \\ College Station, TX 77843 \\ Email: murphy@cse.tamu.edu
}

\author{
Alexander Kleiner \\ Computer and Information Science \\ Linköping University \\ SE-581 83 LINKÖPING SWEDEN \\ Email: alexander.kleiner@liu.se
}

\begin{abstract}
The IEEE Safety, Security, and Rescue Robotics community has created a roadmap for producing unmanned systems that could be adopted by the Public Safety sector within 10 years, given appropriate $R \& D$ investment especially in human-robot interaction and perception. The five applications expected to be of highest value to the Public Safety community, highest value first, are: assisting with routine inspection of the critical infrastructure, "chronic emergencies" such as firefighting, hazardous material spills, port inspection, and damage estimation after a disaster. The technical feasibility of the applications were ranked, with the most attractive scenario, infrastructure inspection, rated as the second easiest scenario; this suggests the maturity of robotics technology is beginning to match stakeholder needs. Each of the five applications were discussed in terms of the six broad enabling technology areas specified in the current National Robotics Initiative Roadmap (perception, human-robot interaction, mechanisms, modeling and simulation, control and planning, and testing and evaluation) and nine specific capabilities identified by the community as being essential to commercialization (communication, alerting, localization, fault tolerance, mapping, manpower needs, plug and play capabilities, multiple users, and multiple robots). The community believes that perception and human-robot interaction are the two biggest barriers to adoption, and require more research, given that their low technical maturity (3rd and 6th rank respectively). However, each of the specific capabilities needed for commercialization are being addressed by current research and could be achieved within 10 years with sustained funding.
\end{abstract}

\section{INTRODUCTION}

In 2012, the IEEE Robotics and Automation Society's Technical Committee on Safety Security and Rescue Robotics (TC-SSRR) reviewed the state of the progress in robots for the Public Safety and created a research and development roadmap to accelerate the adoption of ground, aerial, and marine robots by public agencies. The TC-SSRR was established in 2002, focusing on R\&D with a clear benefit to the Public Safety stakeholders, such as law enforcement, fire rescue, port and border inspection, and environmental protection. It hosts the annual IEEE International Symposium on Safety, Security, and Rescue Robotics (SSRR).

At the 10th annual SSRR in 2012, researchers and developers conducted a a roadmapping exercise with the approximately 100 participants to

- suggest revisions to the US National Robotics Roadmap [1], which is primarily driven by homeland defense (military) applications with little mention of homeland security (Public Safety).

- consolidate the lessons learned in the first 10 years of the field, and

- answer the question of "why agencies aren't adopting these technologies?" as the community felt that the technology had matured rapidly, especially in the last five years.

A follow-up survey was emailed to the conference participants and the general SSRR community. The survey consisted of 10 questions based on the town hall session, where five application scenarios and nine cross-cutting capabilities were identified that were needed to enable a municipality or agency to purchase a robot. $\mathrm{T} 27$ respondents ranked the the scenarios and capabilities by usefulness for an agency and by the degree of difficulty, projected availability, and technical maturity. These scenarios were also rated in terms of the six key enabling technologies defined in the US National Robotics Roadmap.

The 10 questions were:

1) Rank the scenarios as the most useful for a municipality or city ( 1 to 5 , for 5 scenarios, with 1 being most useful)

2) Rank the scenarios in terms of technical challenges (1 to 5 , for 5 scenarios, with 1 being the most technically challenging)

3) Do you wish to propose a scenario? (essay)

4) Rate each of these [nine cross-cutting capabilities] in terms of their importance to creating commercial SSRR robots that will be adopted by responders. (extremely important, definitely needed, probably important in the future, not very important)

5) Rank each of these [nine cross-cutting capabilities] in terms of their technical difficulty. (nearly impossible, problem isn't fully understood, promising solutions are being explored, already exists, just need tech transfer)

6) Rate the key enabling technologies as to whether they are a barrier preventing progress towards commercial systems adopted by the Public Safety sector or would be nice but not essential. (barrier to adoption, barrier to R\&D but users don't care, promising solutions are being explored, solved or not important) 
7) Rank the key enabling technologies on how maturity or sufficient they are. (1-6, for 6 key enabling technologies, with 1 being the most mature)

8) Estimate when these challenges are likely to be solved. (in 5 years, in 10 years, in 15 years)

9) Which region is leading in investment and use of robot for safety, security, and rescue? (1-9, for 9 regions, with 1 being leading)

10) What other technical challenges do you see as critical to SSRR? (essay)

\section{SCENARIOS}

Survey questions 1 and 2 asked respondents to rank the five application scenarios in order of value to a municipality or agency, summarized in Figure 1. The scenarios are described in more detail in the following subsections. Survey question 3 produced two other scenarios: "emergency medical rescue of survivors" and "Urban fires are a very challenging search and rescue scenario whre robotic technology could help much first responders to face them with increased safety and efficacy." It was not clear how the latter scenario differed from the chronic emergency scenario.

The highest value scenario using the average ranking is routine infrastructure inspection (avg. 1.96) by UGVs, UAVs, and UMVs of the critical infrastructure, such as bridges, roads, pipelines, electrical grid, ports, etc. The second is for chronic emergencies (avg. 2.11), as opposed to rare disasters, such as structure fires, hostage negotiations, building collapses, bomb disposal, and SWAT, where UGVs and UAVs can work in front of the responders to provide video, map the actual situation, and sense dangerous conditions; work beside responders to carry equipment and to allow telepresence of a buddy to keep a lookout; and work behind responders to act as network repeaters and emergency exit beacons. The third scenario is hazmat (avg. 3.33), hazardous materials spills such as a chemical train derailment or tanker truck accident, where UGVs and UAVs can approach the source and provide multi-sensor perception of the state of the event, types of chemicals, and if the spill is moving towards high occupancy areas or into sensitive waterways. The fourth scenario is port inspection (avg. 3.63) where UMVs continually inspect pilings and substructures for changes while UAVs and UGVs cover the superstructure. The fifth is damage assessment (avg. 3.96) or estimation of damage after a disaster, thereby speeding up insurance claims, re-entry of homeowners, small business loans, and debris removal.

As shown in Figure 2, the most attractive scenario, infrastructure inspection, was viewed as relatively easy, coming in at 4th place. This might suggest that SSRR robots reside in a development or implementation phase, rather than still undergoing research. But the second most attractive scenario, chronic emergencies, was ranked as the most technically challenging; this ranking suggests that robots still require significant research and development. The success of the robot from one scenario does not mean the technology is mature to handle all scenarios are all hazards.
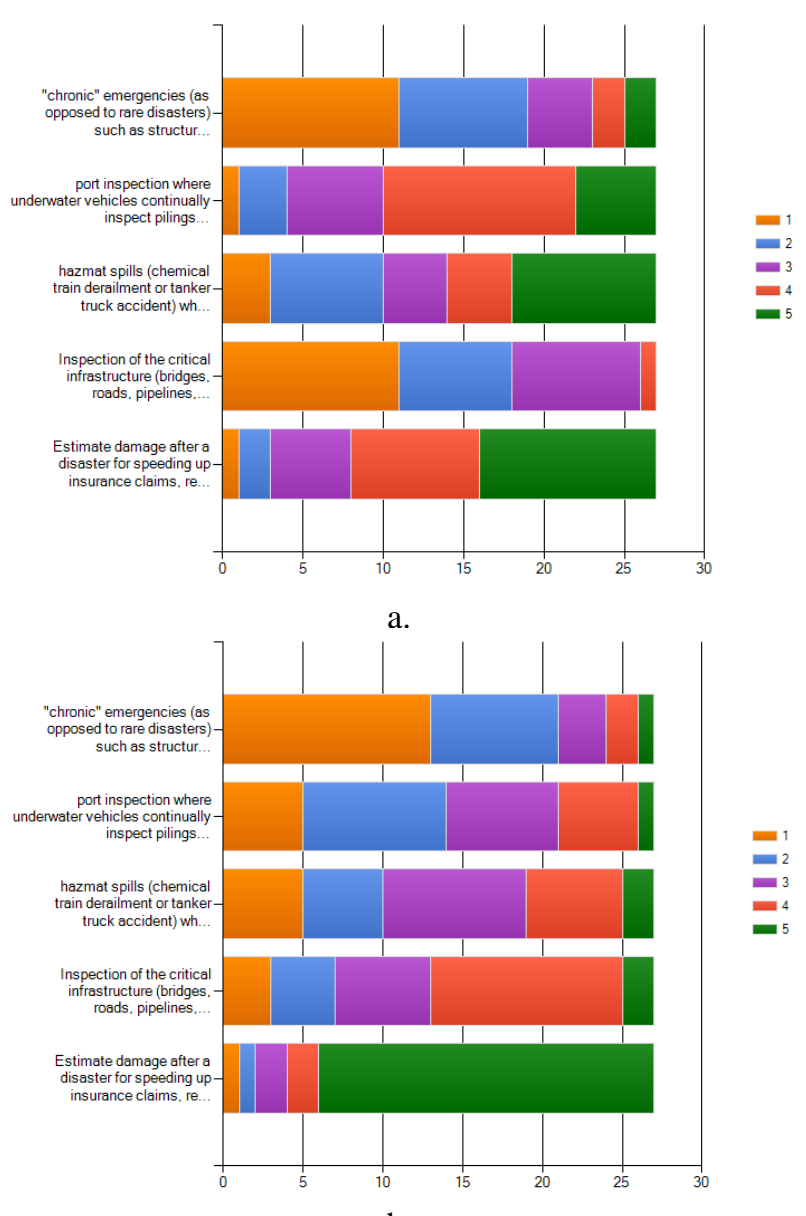

b.

Fig. 1. The five scenarios rankings in order of a) the most useful for a municipality or city; 1 is the most useful, and b) the most technically challenging.

\section{Most Valuable Scenario}

1. Infrastructure inspection

2. Chronic emergencies

3. Hazmat

4. Port Inspection

5. Damage Assessment

\section{Technically Challenging}

1. Chronic emergencies

2. Port Inspection

3. Hazmat

4. Infrastructure inspection

5. Damage Assessment
Fig. 2. Comparison of the ranked usefulness of a robot system with the ranked perceived technical challenges of creating that system.

\section{A. Infrastructure Inspection}

Inspection of the critical infrastructure (bridges, roads, pipelines, electrical grid, ports, etc.) is expected to be of the most immediate and pervasive value to the Public Safety sector. This scenario includes both routine and post-disaster inspections.

Robots add two new capabilities to infrastructure inspection tasks. One is that robots can perform routine inspection while in service without draining pipelines, restricting traffic, etc. 
Ground robots can be used for underground utilities such as buried tunnels of wires and pipes. This is more cost-effective than the current "take it out of service and inspect" methods where internal pipeline or sewage inspection requires utilities to be shut down, bridges to be closed, and access restricted to ports. Second, the robots that are used in daily routine inspection can be inserted for post-disaster inspection such as after a hurricane or earthquake; this dual use provides increased capability, is cost effective, and accelerates economic recovery.

Infrastructure inspection scenarios require reliable localization so that the damaged area is precisely known, the ability for responders to see that true state of the leak or lack of a leak without false positives or negatives, low bandwidth communication to keep up with the heartbeat of the vehicle but to alert the operator if there is a problem, and fault tolerance or graceful degradation of performance. If aerial vehicle are used, the should be reliable in outdoor weather conditions including fog, a wide range of temperatures and presence of shadows due to the time of day, plus be able to function in extraordinary conditions such as at night or in smoke from wildfires.

\section{B. Chronic emergencies}

Assisting with "chronic" emergencies (as opposed to rare disasters) such as structure fires, hostage negotiations, building collapses, bomb disposal, and SWAT was ranked as the second most useful scenario. Robots add three new capabilities for chronic emergencies. They can work in front of the responders to provide video, map the actual situation, and sense dangerous conditions. This would allow the responders to determine the extent of the situation and the degree of personnel required and their level of protection. Robot can also beside responders to carry equipment and to allow telepresence of a buddy to keep a lookout Finally, robots can operate behind responders to act as network repeaters and emergency exit beacons.

Chronic emergency scenarios require that robots have realtime real reliable real-time communication, the ability to sense at night, and through smoke, operate in wet and icy conditions, possibly even have self-cleaning sensors to continue functionally. Reliable localization is needed as well. Human robot interaction should allow multiple users to simultaneously view data from the robot, for example the incident commander, a structural engineer, and the mayor may want to view the data feed at the same time but for different purposes. Chronic emergencies may also require the robot to have dexterous manipulation to open doors and turn valves. Responders in these events want to able to plug-and-play the sensors that they commonly use for these task rather than rely on rather generic sensors provided by robot manufacturer. The robot must be fault tolerant or degrade gracefully, such as having a selfrighting capability and return to home function. Manpower and training are always key issues in the public safety sector and thus human robot interaction techniques which reduce main parent training are desirable. The robot should alert or queue the user to situations or objects of interest but without false positives or negatives.

\section{HazMat}

Responding to hazardous material events, such as a chemical train derailment or tanker truck accident, was ranked third in value. In this scenario a ground, aerial, or marine robot can approach the source and provide multi-sensor perception of the state of the event, the types of chemicals either by finding the labels and placards or sampling the spill, and if the materials are moving towards high occupancy areas or into sensitive waterways. Hazmat events are relatively frequent, with over 32,000 reported instances in 2011 [2], and over 500 of these were significant enough to have injuries or require evacuation or shut down a major transportation route [3].

Robots provide three new capabilities: providing accurate information while the responders are suiting up thus speeding up the response and reducing the impact of the spill, giving the Hazmat incident commander the ability to have direct situation awareness, and reducing the number of experts needed to respond and reducing their exposure and risk.

Hazmat scenarios require advances in human-robot interaction, especially interfaces that tailored to the specific user role, and allow data from the robot to be distributed to multiple users. The robot should display the state of the robot and the ability to conduct the mission, such as that the charge in the batteries are not sufficient for stair climbing, lighting/temp/terrain out of design specs, etc. The data coming from the robot should be distributed based on priority, security, and the ability of role to tolerate lag. Sensing, localization, and mapping are critical as the responders need to know the extent of the spill. The robot must be able to work at night and in extreme conditions of weather, low $\mathrm{pH}$, radiation, and explosive atmospheres.

\section{Port Inspection}

Using robots for port inspection was ranked fourth, where underwater vehicles continually inspect pilings and substructures for changes while ground and aerial vehicles cover the superstructure. The type of ports include cruise ships terminals and piers along public buildings such as the Manhattan, Miami and Chicago River fronts, not just deep water shipping or military ports. After further consideration, the consensus was that the Port Inspection scenario was essentially identical to the Infrastructure Inspection scenario, with the primary difference being the agencies that would use the unmanned systems.

\section{E. Damage Assessment}

Robots can help estimate damage after a disaster, thereby speeding up the filing and accuracy of insurance claims, re-entry of homeowners, small business loans, and debris removal. The consensus was that the damage assessment scenario was essentially identical to the chronic emergencies scenario.

\section{Cross-Cutting CAPABilities NeEded for ADOPTION}

During the conference town hall session, the 100 participants identified nine cross-cutting capabilities as being essential for adoption by the Public Safety sector. The participants 


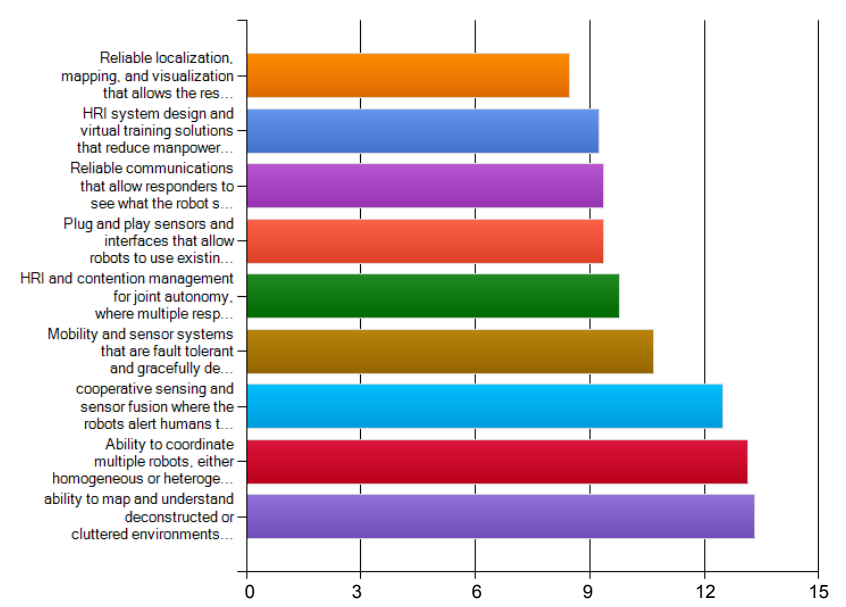

Fig. 3. Projected availability of the nine cross-cutting capabilities arranged by increasing remaining years of $\mathrm{R} \& \mathrm{D}$ (average responses).

did not include privacy or security issues as the consensus was these were policy issues not technical barriers. This should not be construed to mean that the community felt that privacy and security were unimportant or that satisfactory technologies exist for all privacy and security needs, rather privacy and security are highly dependent on the application or agency. After the conference, the 27 survey respondents ranked these in terms of their importance to created creating commercial robots that would be adopted by responders and when the cross-capabilities were be available, either within 5,10 , or 15 years. The results are summarized in Fig. 3.

The cross-cutting capabilities are presented below in order of averaged decreasing importance to commercialization (question 4) with the expected time to availability (question 8):

- Communications 9.36 years. Reliable communications that allow responders to see what the robot sees in real time and direct the robot is needed.

- Alerting 12.48 years. Cooperative sensing and sensor fusion where the robots alert humans to changes or potential danger that don't produce false negatives that increase responder risk

- Fault tolerance 10.68 years. Mobility and sensor systems that are fault tolerant and gracefully degrade.

- Reliable Localization 8.46 years. Reliable localization, mapping, and visualization that allows the responders to comprehend where the robot is.

- Mapping at night and extreme conditions 13.32 years. Ability to map and understand deconstructed our cluttered environments under noise in conditions (nighttime, smoke, turbidity etc.).

- HRI for reducing manpower 9.24 years. HRI system design and virtual train solutions that reduce manpower and training expenses

- Plug-and-play 9.36 years. Plug-and-play sensors and interfaces that allow robots to use existing sensors and tools without a municipality having to buy more (or different)
Biggest Barrier
Maturity of Technology
1. HUMAN-ROBOT INTERACTION
1. MECHANISMS
2. Perception
2. CONTROL AND PLANNING
3. Control and Planning (tied)
3. Perception
4. Test and Evaluation (TIED)
4. Modeling ANd Simulation
5. MECHANISM
5. HUMAN-ROBOT INTERACTION
6. MOdELING \& SIMULATION
6. TESTING AND EVALUATION

Fig. 4. The six key enabling technologies from the NRI Roadmap rated in terms of barriers and maturity; 1 is the most severe barrier or most mature technology.

sensors.

- HRI for multiple users 9.78 years HRI and contingency management for joint autonomy, allowing multiple responders throughout the command echelon to independently, but simultaneously, use a robot to see and act as a distance.

- Coordination of multiple robots 13.14 years. Ability to coordinate multiple robots, either homogeneous or heterogeneous, working cooperatively on different task.

\section{Enabling Technologies Required to CREATE ADOPTABLE SySTEMS}

The follow survey asked respondents were to rate the state of art in terms of six key enabling technology areas specified in the National Robotics Initiative Roadmap: PERCEPTION, HUMAN-ROBOT INTERACTION, MECHANISMS, MODELING AND SIMULATION, CONTROL AND PLANNING, and TESTING AND EVALUATION. Question 6 asked whether each of the six technologies were a barrier preventing progress toward commercial systems adopted by the public safety sector or whether they were desirable but not essential, while question 7 asked what was the current level of maturity or sufficiency of each of the technologies. Figure 4 captures and compares the results. The results suggest that progress along the National Robotics Roadmap will be beneficial in general to the SSRR community.

The survey indicated the biggest barriers to adoption were HUMAN ROBOT INTERACTION and PERCEPTION. CONTROL AND PLANNING was tied with TEST AND EVALUATION, followed by MECHANISMS and MODELING AND SIMULATION. Unfortunately as can be seen by in Fig. 4 the two biggest barriers, HUMAN ROBOT INTERACTION and PERCEPTION, have a relatively low maturity with PERCEPTION ranking third and HUMAN ROBOT INTERACTION fifth. This suggests that adoption requires more research and development in human robot interaction and perception period.

\section{OTHER OBSERVATIONS}

The community identified other technical challenges during the follow-up survey's open ended question "What other technical challenges do you see as critical to SSRR?" Three respondents reported "Portability, ease of maintenance of (broken) robot systems in the field, mobile command post," 
"Partial autonomy of robots," and "Autonomy, endurance, size, cost and shielding from radiation and explosive proof." These responses suggest that the NRI and the SSRR community may be under-estimating the importance of practical attributes of the robots.

The community was asked to rate itself in terms of who is leading in the development and use of safety security and rescue robots. During the conference, the participants agreed upon 9 geographical areas; the follow-up survey asked the participants to rank the 9. The survey respondents reported North America (1.93 average rating) led followed by Japan (2.48), the European Union (3.33), China (4.89), Australia (4.93), Korea (5.26), Latin and South America (6.85), Africa (7.04), and other (8.30). This was a subjective rating which was not based on any analysis of the economic development, but rather upon collective perception of funding for SSRR research and development.

\section{CONCLUSIONS}

The Safety, Security, and Rescue Robotics community believes that robots could be mainstreamed into the Public Safety section within 10 years, given appropriate $R \& D$ investment, especially in HUMAN-ROBOT INTERACTION and PERCEPTION. The need for investment in HUMAN-ROBOT INTERACTION and PERCEPTION is consistent with the National Robotics Initiative Roadmap. The SSRR community believes there is a market for robot systems that can be used for "routine" inspection, such as for critical infrastructure, and "chronic emergencies," such as fires and chemical spills. Ideally, these robot systems would provide a basis for extending capabilities needed for hazmat response and port inspection, and could be re-tasked for damage assessment after a disaster. The barriers to adoption are not the mechanical agility of the robots but rather the lack of human-robot interaction principles that would make it easy to use the robots without extensive training or high cognitive loads. The community discussion noted that human-robot interaction styles for public safety stakeholders is quite different than for military users. The lack of reliable sensors and algorithms for sensing is another key barrier. The survey indicates that the community believes the US leads in R\&D, but the discussion at the conference suggested that the US is not leading in applications of safety, security, and rescue robots. Japan, European Union, and China are perceived as actively pursuing these areas and may have more favorable regulations and funding mechanisms that foster adoption of robots by the Public Safety sector.

\section{ACKNOWLEDGMENTS}

The author would like to thank the SSRR community for their responses and the anonymous reviewers.

\section{REFERENCES}

[1] "A roadmap for u.s. robotics: From internet to robotics, robotics caucus advisory committee," Tech. Rep., 2013.

[2] USCG, "National response center: Statistics incident type 2000-2011," 2012.

[3] globalincidentmap.com, "North american hazmat situations and deployments map," 2012. 